\title{
Implication des corps \\ nucléaires PML \\ dans l'immunité \\ intrinsèque et innée
}

> PML (promyelocytic leukemia), appelée aussi TRIM19 (tripartite motif protein 19), est une protéine impliquée dans l'organisation des corps nucléaires PML (CN PML). Ces structures multiprotéiques sont associées à la matrice nucléaire; elles recrutent un grand nombre de protéines et sont impliquées dans divers processus cellulaires, dont la défense antivirale. La conjugaison de PML à SUMO (small ubiquitin modifier) est requise pour la formation et la fonction des CN PML. Plusieurs isoformes de $P M L$ sont exprimées à partir d'un gène unique; toutes possèdent une région carboxy-terminale spécifique, qui leur permet d'interagir avec des partenaires différents et leur confère ainsi des fonctions propres. Certaines sont douées d'une activité antivirale, permettant à PML de jouer un rôle clé dans l'immunité antivirale intrinsèque. PML est aussi un important régulateur de la réponse interféron déclenchée lors d'une infection virale. Dans cette revue, nous discutons l'implication des isoformes de PML dans la réponse interféron et la défense antivirale, à l'interface des immunités intrinsèque et innée. <

La première ligne de défense contre les agents pathogènes est constituée, outre les barrières physiologiques, par l'immunité innée [38]. L'immunité innée consiste en la détection de composants de ces pathogènes, signatures moléculaires désignées pathogen-associated molecular patterns (PAMP), par des détecteurs cellulaires connus sous le nom de pattern-recognition receptors ( $P R R)$ [39]. En réponse à la détection d'un PAMP, ces PRR déclenchent des signaux de transduction qui, à leur tour, sont responsables d'une réponse adaptée à l'élimination du pathogène. Dans le cas des virus, il s'agit de la réponse interféron (IFN).

Par ailleurs, l'existence d'autres mécanismes de défense aux infections virales, impliquant des facteurs antiviraux cellulaires désignés sous le terme de facteurs de

Mohamed Ali Maroui, Faten El Asmi, Jacques Dutrieux, Mounira K. Chelbi-Alix, Sébastien Nisole

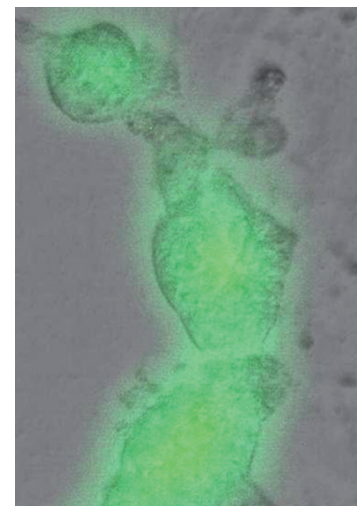

Inserm UMR-S 1124, université Paris Descartes, 45 , rue des Saints-Pères, 75006 Paris, France.

sebastien.nisole@inserm.fr

restriction, a été recemment identifiée ; cette protection cellulaire a été qualifiée d'immunité intrinsèque [1]. Cependant, comme l'expression de ces facteurs est aussi induite par l'IFN, la frontière entre ces deux types d'immunité est relativement floue.

La famille des protéines TRIM (tripartite motif) comprend plus de 70 protéines chez l'homme. Ces protéines se caractérisent par une organisation en trois domaines : un domaine RING, une ou deux $B-B o x$ et un domaine coiled-coil ; ce motif tripartite est ainsi désigné RBCC. Ces protéines TRIM ont récemment été identifiées comme des acteurs clés de la défense antivirale. Ainsi, certaines d'entre elles possèdent une activité antivirale intrinsèque, TRIM5a, TRIM19 ou TRIM22, tandis que de nombreuses autres régulent de façon importante les voies de signalisation qui déclenchent la synthèse d'IFN et/ou de cytokines pro-inflammatoires [2-4].

La protéine TRIM19, mieux connue sous le nom de PML (promyelocytic leukemia), a tout d'abord été identifiée sous forme d'une protéine de fusion PML-RAR $\alpha$ entre PML et le récepteur de l'acide rétinoïque (RAR- $\alpha$ ), conséquence d'une translocation chromosomique $t(15 ; 17)$ intervenant chez des patients atteints de leucémie aiguë promyélocytaire [5]. Du fait d'un épissage alternatif à partir d'un gène unique, il existe sept isoformes de PML (PMLI à VIIb) ayant toutes le motif RBCC/TRIM, mais une extrémité carboxy-terminale spécifique (Figure 1) $[6,7]$. La variabilité de la région carboxy-terminale des isoformes de PML leur permet d'interagir avec différents partenaires et leur confère donc des fonctions spécifiques [6]. PML a une 

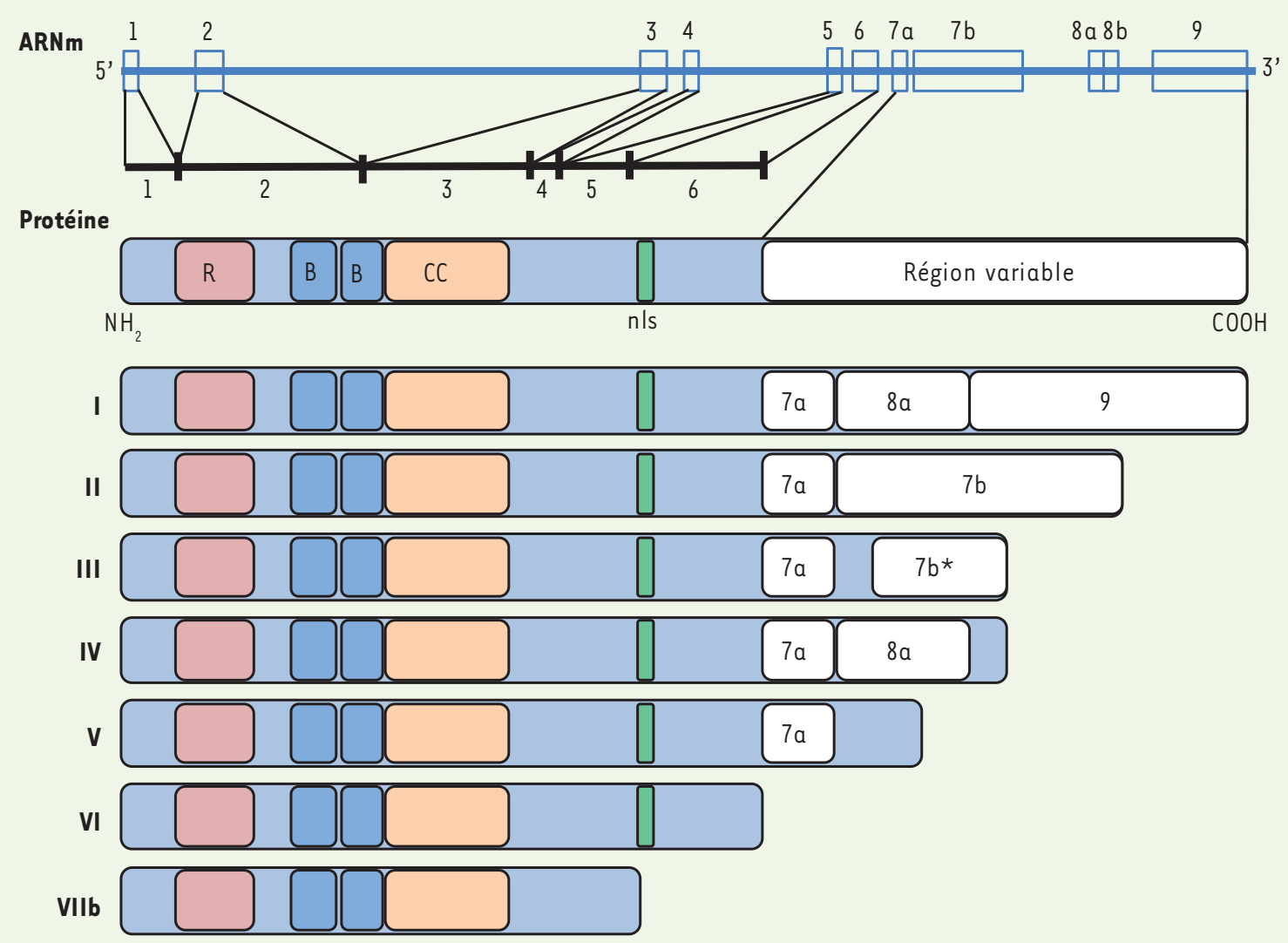

Figure 1. Génération des sept principales isoformes de PML. Le gène PML est constitué de neuf exons (notés de 1 à 9 ), les exons 7 et 8 étant subdivisés en exons $7 a, 7 b, 8 a$ et $8 b$. L'épissage alternatif du transcrit PML conduit à la traduction de sept isoformes, désignées PMLI à PMLVIIb. Toutes les isoformes possédent le motif RBCC/TRIM en amino-terminal et une région carboxy-terminale spécifique. La composition en exons de la portion carboxy-terminale de chaque isoforme est représentée. L'exon $7 \mathrm{~b}$ * présent dans l'isoforme PMLIII est hors cadre de lecture, ce qui génère un codon STOP précoce. Les isoformes PMLI à PMLVI, toutes pourvues du signal de localisation nucléaire (nls), sont nucléaires, tandis que l'isoforme PMLVIIb, qui en est dénuée, est strictement cytoplasmique.

distribution intranucléaire, avec une forme diffuse majoritaire dans le nucléoplasme et une forme associée à la matrice nucléaire appelée en français corps nucléaire PML (CN PML) et en anglais PML nuclear body (PML NB) (Figure 2). Les CN PML sont des structures nucléaires multiprotéiques dont PML est «l'organisatrice» [8]. Ces structures contiennent des protéines résidentes de façon permanente, comme PML, Sp100 et SUMO (small ubiquitin modifier), et une multitude de protéines y sont recrutées de façon transitoire et en réponse à divers stimulus (Figure 2). PML se lie à SUMO de façon covalente (processus appelé SUMOylation), modification qui régule sa localisation subcellulaire, sa stabilité, sa capacité à former les CN PML et à recruter différents partenaires au sein de ces structures. SUMO peut également se fixer à PML de façon non covalente, via le domaine SIM (SUMO interacting motif) de certaines isoformes de PML, domaine situé au niveau de leur extrémité carboxy-terminale (Figure 2). PML et les CN PML sont impliqués dans plusieurs processus cellulaires tels que l'apoptose, la sénescence, la différenciation cellulaire, ainsi que la stabilité du génome [9-12]; notre groupe a contribué à montrer leur implication dans la réponse à l'IFN et la défense antivirale [6, 13-21] (Figure 2).

Dans cette revue, nous résumons les différentes études qui ont permis d'identifier PML comme un important acteur des immunités antivirales intrinsèque et innée. Les virus dont il sera question ici appartiennent à différentes familles virales, à ADN ou à ARN. Parmi les virus à ARN, nous citerons des membres de la famille des Picornaviridae (virus de l'encéphalomyocardite, EMCV), des Rhabdoviridae (virus de la rage et virus de la stomatite vésiculaire, VSV), des Retroviridae (virus T-lymphotrope humain de type I, HTLV-1), des Orthomyxoviridae (virus de la grippe), des Paramyxoviridae (virus Sendaï, SeV); les virus à $A D N$ mentionnés comprennent les Herpesviridae (virus varicelle-zona, VZV) et les Poxviridae (virus de la vaccine). 

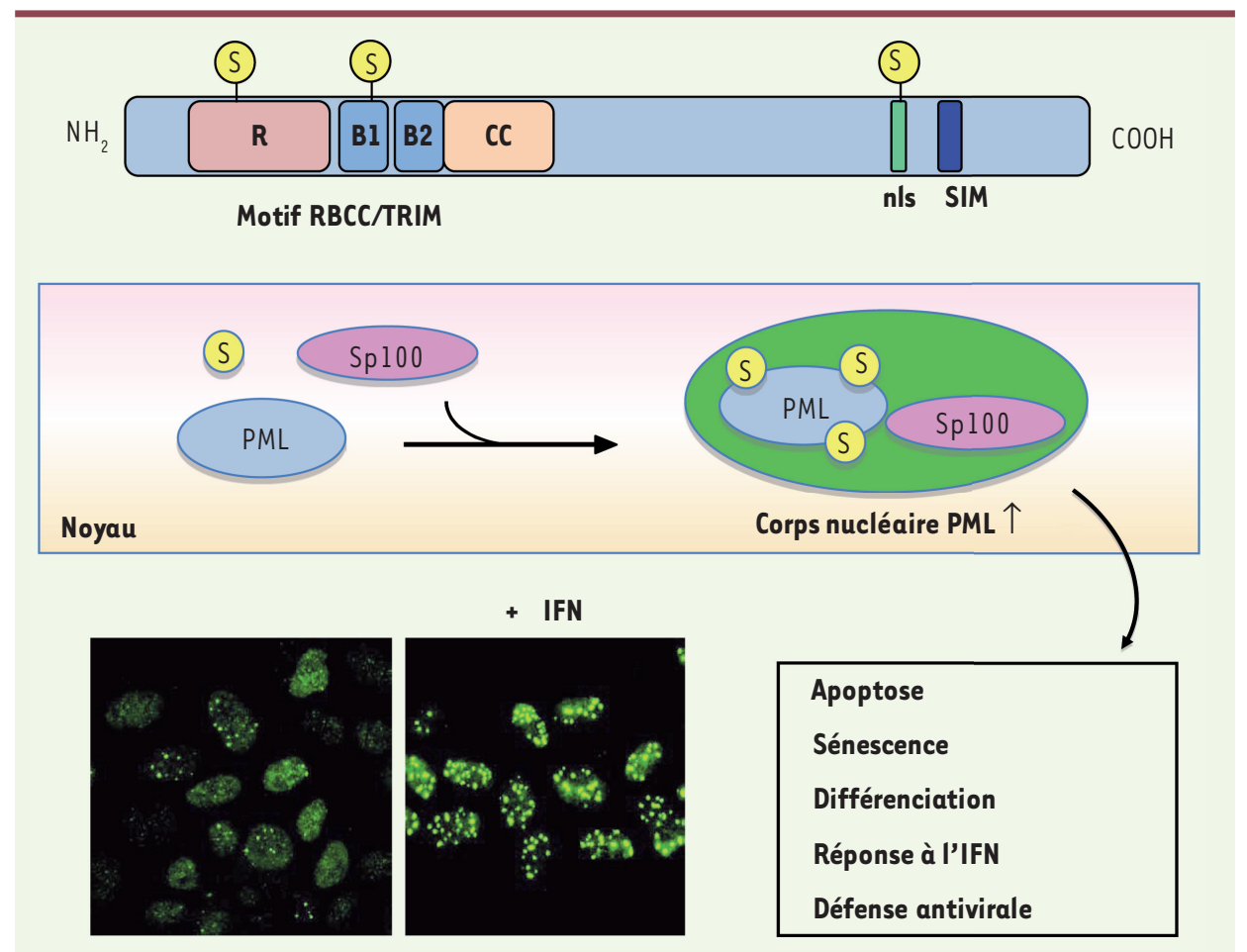

Figure 2. PML est l'organisatrice des CN. PML peut être conjuguée à SUMO (représenté par un « $\mathrm{S}$ ») au niveau de trois résidus lysine situés dans le domaine RING ( $R$ ), dans le domaine B-Box 1 ( $B l$ ) et au niveau du nls. La conjugaison de PML à SUMO est requise pour la formation de CN PML fonctionnels. Les isoformes PMLI à $V$ possèdent également un domaine SIM (SUMO interacting motif), qui permet la fixation non covalente de SUMO. Outre SUMO et PML, Spl00 est également une protéine résidente des CN PML. PML et les CN PML sont impliqués dans plusieurs processus cellulaires tels que l'apoptose, la sénescence, la différenciation cellulaire, la réponse à I'IFN et la défense antivirale. PML a une distribution intranucléaire avec une forme diffuse majoritaire dans le nucléoplasme et une forme asso-

ciée aux CN PML détectée sous forme de spots nucléaires par immunofluorescence. L'IFN induit directement l'expression du gène de PML, et augmente le nombre et la taille des CN PML.

\section{L'expression de PML/TRIM19 est directement induite par l'interféron}

Les interférons (IFN) ont été découverts en 1957 pour leur propriété antivirale. Depuis cette date, d'autres activités leur ont été attribuées, telles que l'inhibition de la multiplication cellulaire et l'induction de l'apoptose, mais c'est toujours l'activité antivirale qui permet de les définir. Ils sont utilisés dans le traitement de maladies virales et en cancérologie. Les IFN sont classés en trois types : I ( $\alpha$, $\beta$ et $\omega)$, II $(\gamma)$ et III $(\lambda)$ [22]. Après fixation des IFN de type I sur leurs récepteurs (IFNARI/IFNAR2), les protéine kinases JAK1 (Janus kinase 1) et TYK2 (tyrosine kinase 2) sont phosphorylées, et phosphorylent à leur tour les protéines STAT (signal transducer and activator of transcription, STATl et -2), qui se lient alors à IRF-9 (interferon regulatory factor-9). Ce complexe migre vers le noyau, se fixe sur une séquence d'ADN dite ISRE (interferon-stimulated response element) et active la transcription des gènes répondant aux IFN de type I. Sous l'effet de I'IFN de type II (IFN $\gamma$ ), les kinases JAKI et -2 sont activées, et elles phosphorylent ainsi STATl, ce qui aboutit à la formation d'homodimères. Ceux-ci migrent vers le noyau, se fixent sur une séquence GAS (gamma activation site) et activent la transcription des gènes répondant à I'IFN de type II. Les IFN de type III, bien qu'ils se fixent à des récepteurs spécifiques, activent la même voie de signalisation que l'IFN de type I et régulent donc l'expression des mêmes gènes.
PML/TRIM19 est la première des protéines TRIM identifiées dont l'expression est augmentée dans les cellules traitées par l'IFN [23]. En effet, l'expression du gène $P M L$ est directement induite par tous les types d'IFN, via les séquences ISRE (-GAGAATCGAAACT-) et GAS (-TTTACCGTAAG-) présents dans son promoteur [24]; cet effet aboutit à l'augmentation de l'expression des différentes isoformes de PML et à l'augmentation des CN PML, en nombre et en taille [23]. Les IFN induisent l'expression des gènes codant pour des protéines relayant leurs effets biologiques. PML, l'organisatrice des CN PML, est l'une de ces protéines, et elle joue un rôle clé dans la réponse à I'IFN. De plus, PML régule positivement le signal de transduction de l'IFN de type II en prolongeant l'activation de STATl et sa liaison à la séquence GAS, augmentant ainsi la réponse biologique à cet IFN [25].

\section{Implication de PML/TRIM19 dans l'activité antivirale intrinsèque}

PML/TRIM19 exerce une activité antivirale intrinsèque vis-à-vis de virus appartenant à des familles différentes $[6,13,14,19-21]$. Le rôle de PML dans la défense antivirale est aussi attesté par le fait que les souris invalidées pour le gène $\mathrm{pml}$ sont plus sensibles aux 


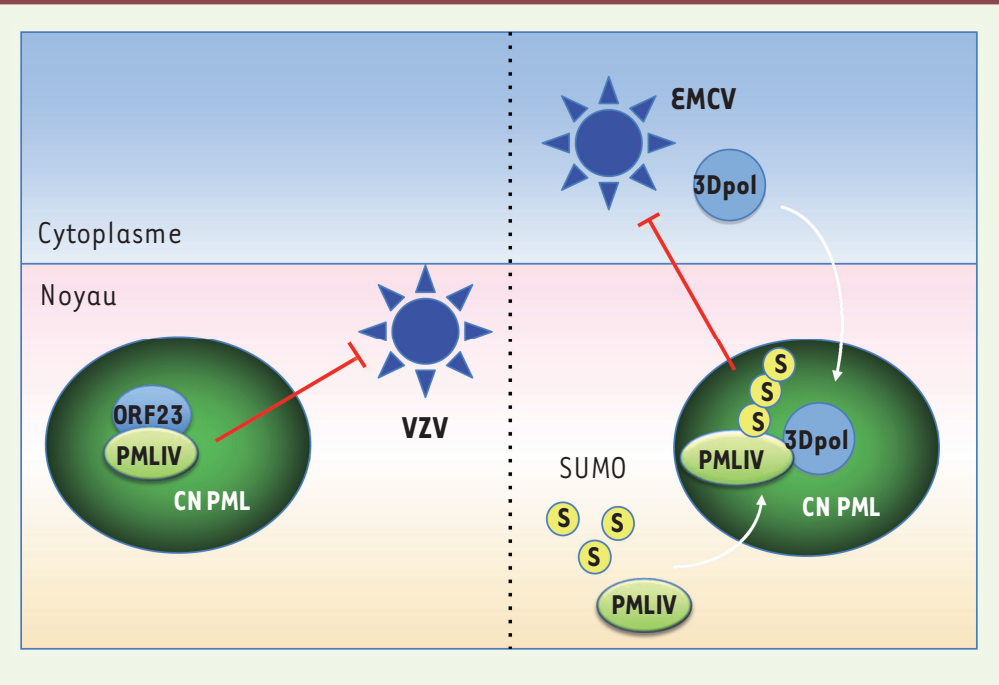

Figure 3. PMLIV confère une résistance au VZV et à l'EMCV. Comme celle de tous les virus de la famille des Herpesviridae, la réplication du VZV se déroule dans le noyau des cellules infectées. L'EMCV appartient quant à lui à la famille des Picornaviridae et se réplique exclusivement dans le cytoplasme. Seules les protéines virales $3 \mathrm{Dpol}$ et 3 Cpro se retrouvent dans le noyau au début de l'infection. Parmi toutes les isoformes de PML, seule PMLIV est capable de séquestrer au sein des CN PML la capside ORF23 du VZV et la 3Dpol de l'EMCV, inhibant ainsi la multiplication de ces virus.

interférence rend les cellules plus sensibles à l'infection [33] suggèrant le rôle de PML/ TRIM19 dans la défense antivirale vis-à-vis du VZV. Comme pour les cellules infectées par

infections virales que les souris parentales [26]. Certaines isoformes de PML ont été identifiées comme capables de conférer une résistance à des virus à $A R N$ ou à $\operatorname{ADN}[6,15-21,27-30]$. PMLIV, notamment, possède une activité antivirale intrinsèque vis-à-vis de l'EMCV [17], du virus de la rage [16], du VZV [28] et du VSV [13, 18, 26]. Nous ne développerons ici que les effets de PMLIV sur l'infection par l'EMCV et le VZV, virus qui se répliquent respectivement dans le cytoplasme et dans le noyau. Dans les deux cas, PMLIV interagit avec une protéine virale, la séquestre au sein des CN PML, inhibant ainsi ses fonctions et, par conséquent, la multiplication virale (Figure 3).

L'EMCV appartient à la famille des Picornaviridae et au genre Cardiovirus. Son génome est un ARN simple brin de polarité positive. Bien que l'EMCV se réplique entièrement dans le cytoplasme, la protéine virale 3D polymérase (3Dpol) et la protéase virale 3Cpro se retrouvent dans le noyau au début de l'infection, formant des structures ponctuées [17, 31]. Les fibroblastes embryonnaires qui dérivent des souris invalidées pour le gène $\mathrm{pml}\left(\mathrm{MEF} \mathrm{PML}^{-/-}\right)$sont plus sensibles à l'infection par l'EMCV que les MEF parentaux [32], suggérant l'implication de PML dans la défense contre ce virus. Une étude surexprimant chacune des isoformes de PML (PMLI à PMLVIIb) d'une manière stable dans des cellules humaines a montré que seule PMLIV protège les cellules de l'infection par L'EMCV. PMLIV, via sa région spécifique carboxy-terminale, interagit avec la polymérase virale 3Dpol et la séquestre dans les CN PML, ce qui affecte la réplication de I'ARN viral [17]. La SUMOylation de PMLIV est requise pour cet effet antiviral puisque le mutant PMLIV3KR non SUMOylable n'est plus capable de recruter la 3Dpol au niveau des CN PML et, par conséquent, n'inhibe plus la réplication virale [17] (Figure 3). Fait remarquable, la diminution spécifique de l'expression de PMLIV par ARN interférence réduit considérablement l'effet protecteur de I'IFN dans les cellules infectées par l'EMCV, démontrant que PMLIV est un médiateur de l'effet anti-EMCV de l'IFN.

Le virus varicelle-zona (VZV) appartient à la famille des Herpesviridae et possède un génome à $A D N$ double-brin linéaire; sa réplication se déroule dans le noyau. La diminution de l'expression de PML par ARN

I'EMCV, seule la surexpression de PMLIV confère une résistance au VZV en séquestrant la protéine de capside ORF23 (open reading frame 23) dans les CN PML [28] (Figure 3). Le domaine carboxy-terminal de PMLIV est requis pour l'interaction avec 0 RF2 3 et sa séquestration dans les CN PML, et, par conséquent, pour l'effet antiviral. En effet, le mutant de PMLIV, PMLIVA8ab, dépourvu de la région carboxy-terminale spécifique de cette isoforme, ne se lie plus à ORF23 et est dénué d'activité antivirale [28].

Ces travaux décryptent les mécanismes par lesquels une isoforme de PML, PMLIV, inhibe de façon intrinsèque un virus à ARN qui se réplique dans le cytoplasme et un virus à $A D N$ qui se réplique dans le noyau, en séquestrant des protéines virales au sein des CN PML.

\section{PML/TRIM19 intervient dans l'immunité innée en ciblant Pin1}

Notre équipe a montré qu'en plus de son activité antivirale intrinsèque vis-à-vis de virus de familles différentes, PMLIV a aussi une activité plus tardive, régulant positivement la production d'IFN- $\beta$ via la séquestration de Pinl dans les CN PML [18].

Pinl est une peptidyl-prolyl cis/trans isomerase (PPlase) qui a tout d'abord été identifiée au cours du criblage de molécules régulant la mitose $[34,40](\rightarrow)$. Pinl est constituée de 163 acides aminés et contient deux domaines fonctionnels: le domaine aminoterminal de liaison WW et le

$(\rightarrow)$ Voir la Synthèse de J. Marsolier et J.B. Weitzman, page 772 de ce numéro domaine carboxy-terminal peptidyl-prolyl isomérase. Le domaine de liaison WW est responsable de la reconnaissance spécifique de protéines phosphorylées au niveau de motifs sérine-proline, tandis que le domaine 


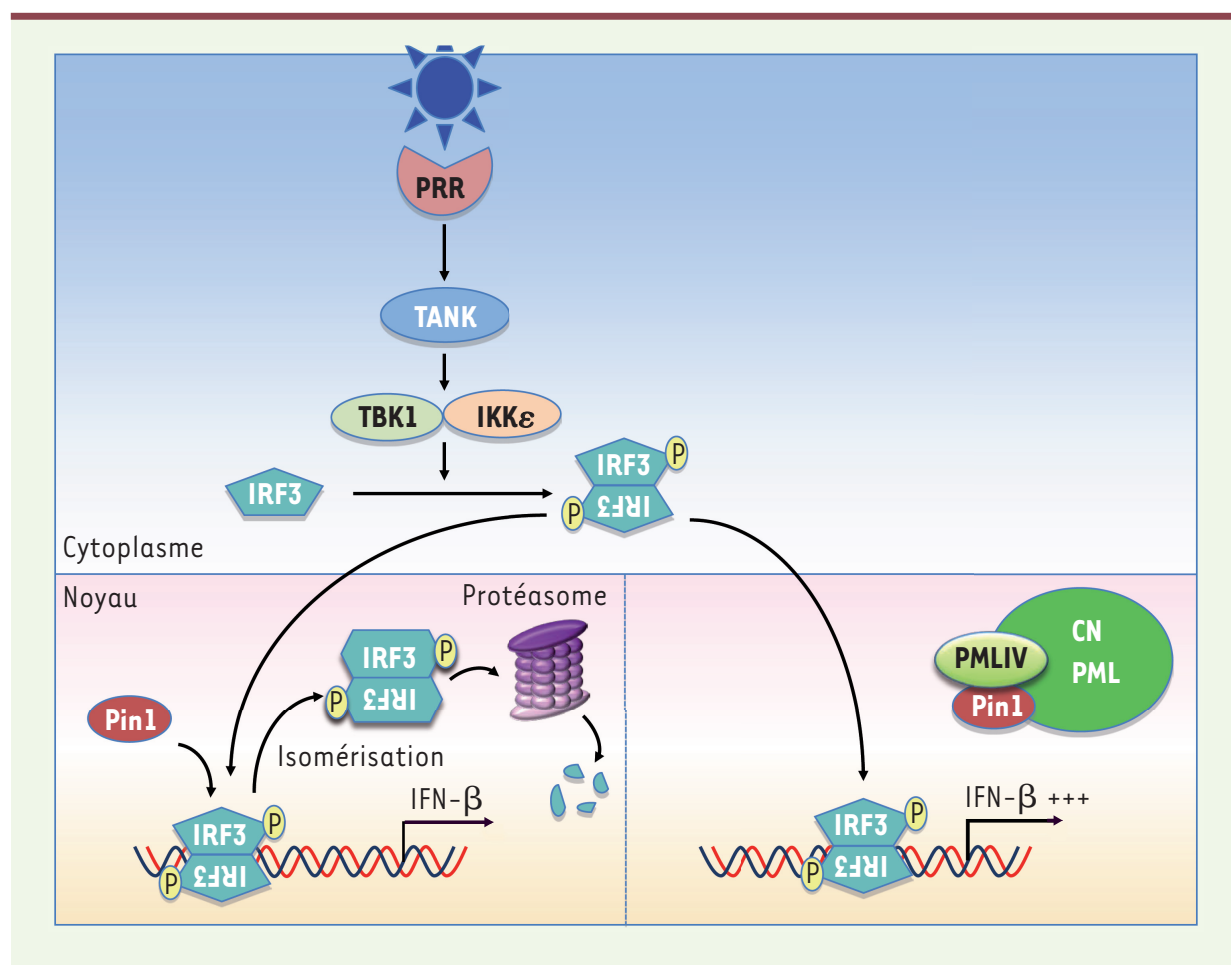

Figure 4. PMLIV stimule la synthèse d'IFN- $\beta$ en séquestrant Pinl. Lorsqu'un virus infecte une cellule, ses composants protéiques ou nucléiques sont reconnus par des détecteurs cellulaires, les PRR (pathogen recognition receptor). Cette détection déclenche une cascade de transduction qui aboutit, quel que soit le virus, à la phosphorylation du facteur de transcription IRF3, à sa dimérisation et à sa translocation dans le noyau, où il induit l'expression d'IFN- $\beta$. Cette activation transcriptionnelle est ensuite arrêtée par l'interaction de Pinl avec IRF3 phosphorylé, qui l'isomérise et entraîne sa dégradation par le système ubiquitine/protéasome. La surexpression de PMLIV entraîne un recrutement de Pinl au sein des CN PML, empêchant ainsi la dégra-

dation d'IRF3 phosphorylé induite par Pinl. II en résulte une forte augmentation de la synthèse d'IFN- $\beta$. IKK : IKB kinase ; TANK : TRAF (TNF receptor associated factor) family member-associated NFkB activator; TBKl : TANK-binding kinase 1.

isomérase carboxy-terminal catalyse l'isomérisation cis/trans du pont peptidique. Ce changement de conformation a des effets importants sur la phosphorylation, la conformation, la localisation subcellulaire, la stabilité et la fonction de nombreux substrats de Pinl [35]. Pinl participe ainsi à la régulation de très nombreux processus cellulaires comme le contrôle du cycle cellulaire, la production de cytokines, la régulation transcriptionnelle, la tumorigenèse ou la neurodégénérescence.

Parmi les nombreuses protéines identifiées comme étant la cible de Pinl figure le facteur de transcription IRF3 (interferon regulatory factor 3) [36]. Comme résumé dans l'introduction, la reconnaissance d'un PAMP viral par l'un des PRR cellulaires induit une cascade de transduction spécifique. Cependant, quel que soit le PRR activé, toutes les voies de signalisation aboutissent à la phosphorylation d'IRF3, suivie de sa dimérisation et de sa translocation dans le noyau. À ce niveau, les dimères d'IRF3 phosphorylé (IRF3-P) induisent la transcription du gène codant pour l'IFN- $\beta$ en se liant aux éléments de réponse situés dans son promoteur. Une fois la réponse IFN enclenchée, le retour à l'état basal est assuré par la reconnaissance du facteur de transcription IRF3-P par Pinl, qui provoque son isomérisation; celle-ci conduit à la dégradation d'IRF3-P par le protéasome [36] (Figure 4).

Nous avons montré pour la première fois que parmi toutes les isoformes de PML, seule PMLIV est un régulateur positif de la production d'IFN en réponse à une infection virale. Plus particulièrement, la surexpression de PMLIV induit une forte augmentation de la transcription d'IFN- $\beta$ lors de l'infection par des virus de différentes familles, à ADN et à ARN ; citons le VSV, le virus Sendaï (SeV), le virus de la grippe,
l'EMCV, le virus de la vaccine ou le HTLV-I. Un tel effet est également observé dans les cellules transfectées avec un analogue synthétique de I'ARN double brin, le poly $(I: C)$. L'augmentation de la synthèse d'IFN- $\beta$ par PMLIV, qui varie de 10 à 100 fois selon le virus, dépend d'IRF3. En effet, nous avons observé que la surexpression de PMLIV conduit à une plus grande stabilité d'IRF3-P dans les cellules infectées. L'IFN- $\beta$ synthétisé produit est capable de protéger d'autres cellules d'une infection ultérieure. À l'inverse, la diminution spécifique de l'expression de PMLIV endogène par ARN interférence dans différentes lignées cellulaires humaines engendre une forte diminution du niveau d'IRF3-P et de la synthèse d'IFN- $\beta$ suite à une infection virale.

Le mécanisme d'action implique une interaction de PMLIV avec Pinl ; celle-ci induit le transfert de Pinl à la matrice nucléaire et son recrutement dans les CN PML. Le recrutement de Pinl dans les CN PML empêche l'isomérisation d'IRF3-P et sa dégradation. La stabilisation d'IRF3-P prolonge la transcription du gène codant l'IFN$\beta$ et donc la synthèse de ce dernier lors d'une infection virale (Figure 4). Il est intéressant de constater qu'une autre protéine de la famille TRIM, TRIM21, a également été décrite comme augmentant la réponse IFN via Pinl. Contrairement à PMLIV, TRIM2l ne séquestre pas Pinl, mais empêche son interaction avec IRF3-P [37]. 
Ces résultats démontrent donc, qu'en plus de ses activités antivirales intrinsèques, PMLIV est également capable de réguler la réponse innée au cours d'une infection virale. Ces travaux suggèrent aussi qu'en recrutant Pinl au sein des CN PML, PMLIV pourrait inhiber d'autres fonctions de Pinl. D’autres études seront nécessaires pour approfondir ces résultats.

\section{Conclusion}

Les CN PML sont, comme les corps de Cajal ou les nucléoles, des structures nucléaires dynamiques, impliquées dans de nombreuses activités biologiques. Bien que leur fonction exacte ne soit pas encore totalement élucidée, les CN PML semblent représenter des plates-formes de rétention, de modification et de dégradation protéique. PML, qui est l'organisatrice de ces corps, est le produit d'un gène dont l'expression est directement induite par l'IFN, et cette induction provoque une augmentation du nombre et de la taille des CN PML. PML exerce une activité antivirale intrinsèque en recrutant des protéines virales au sein des CN PML, inhibant ainsi leur fonction. De nouvelles données montrent que PML régule positivement la production d'IFN de type I dans les cellules infectées par des virus à ARN et à ADN de différentes familles, et que la sumoylation de PML est requise pour ce processus. Ces différentes observations renforcent l'idée selon laquelle les CN PML sont également des centres importants de la défense antivirale et de la réponse IFN. De nombreux virus ont développé diverses stratégies pour contrecarrer la réponse antivirale et utiliser la machinerie cellulaire à leur profit. Ainsi, plusieurs virus induisent la dégradation de PML et/ou sa délocalisation des CN PML. L'altération des CN consécutive à une infection virale bloque l'activité antivirale intrinsèque de PML ainsi que sa capacité à accroître la production d'IFN.

À l'instar de PML/TRIM19, un nombre croissant de protéines de la famille TRIM ont été identifiées comme étant dotées d'une activité antivirale intrinsèque et/ou impliquées dans la régulation de la réponse IFN [2-4]. Le terme de «TRIMmunité », récemment introduit [2], illustre les nouvelles perspectives de recherche concernant cette vaste famille de protéines, très conservée au cours de l'évolution. Des perspectives thérapeutiques peuvent notamment être envisagées, étant donné le positionnement central de ces protéines à l'interface des immunités intrinsèque et innée. $\diamond$

\section{SUMMARY}

Implication of PML nuclear bodies in intrinsic and innate immunity PML/TRIM19 is the organizer of PML nuclear bodies (NB), large multiprotein structures associated to the nuclear matrix, which recruit a great number of proteins and which are implicated in various cellular processes including antiviral defense. The conjugation of PML to SUMO is required for the formation and function of PML NB. Alternative splicing from a single PML gene generates several PML isoforms (PMLI to PMLVIIb), each harboring a specific carboxy-terminal region. This variability allows each isoform to recruit different partners and thus confers them specific functions. $P M L$ gene is directly induced by interferon and certain PML isoforms are implicated in its antiviral properties, as they display intrinsic antiviral activities against RNA or DNA viruses. One isoform, PMLIV, is also impli- cated in innate immunity by enhancing IFN- $\beta$ production during a viral infection. Here we review recent findings on PML/TRIM19 implication in interferon response and antiviral defense, at the interface between intrinsic and innate immunity. $\diamond$

\section{LIENS D'INTÉRÊT}

Les auteurs déclarent n'avoir aucun lien d'intérêt concernant les données publiées dans cet article.

\section{RÉFÉRENCES}

1. Bieniasz PD. Intrinsic immunity: a front-line defense against viral attack. Nat Immunol $2004 ; 5$ : 1109-15.

2. Rajsbaum R, Garcia-Sastre A, Versteeg GA. TRIMmunity: the roles of the TRIM ع3-ubiquitin ligase family in innate antiviral immunity. J Mol Biol 2013; 426: 1265-84.

3. Nisole S, Stoye JP, Saib A. TRIM family proteins: retroviral restriction and antiviral defence. Nat Rev Microbiol 2005 ; 3 : 799-808.

4. McNab FW, Rajsbaum R, Stoye JP, O'Garra A. Tripartite-motif proteins and innate immune regulation. Curr Opin Immunol 2011 ; 23 : 46-56.

5. De Thé H, Lavau C, Marchio A, et al. The PML-RAR alpha fusion mRNA generated by the $\mathrm{t}(15 ; 17)$ translocation in acute promyelocytic leukemia encodes a functionally altered RAR. Cell $1991 ; 66: 675-84$.

6. Nisole S, Maroui MA, Mascle XH, et al. Differential roles of PML isoforms. Front Oncol $2013 ; 3: 125$.

7. Jensen $K$, Shiels $C$, Freemont PS. PML protein isoforms and the RBCC/TRIM motif. Oncogene $2001 ; 20: 7223-33$.

8. Ishov AM, Sotnikov AG, Negorev D, et al. PML is critical for ND10 formation and recruits the PML-interacting protein daxx to this nuclear structure when modified by SUMO-1. J Cell Biol 1999 ; $147: 221-34$

9. Bernardi R, Papa A, Pandolfi PP. Regulation of apoptosis by PML and the PML-NBs. Oncogene $2008 ; 27$ : 6299-312.

10. Krieghoff-Henning $\varepsilon$, Hofmann TG. Role of nuclear bodies in apoptosis signalling. Biochim Biophys Acta 2008 ; 1783 : 2185-94.

11. Jin G, Wang YJ, Lin HK. Emerging cellular functions of cytoplasmic PML. Front Oncol $2013 ; 3: 147$

12. Martin N, Dejean A, Bischof 0 . TBX2, un nouvel acteur dans la sénescence cellulaire induite par PML. Med Sci (Paris) $2012 ; 28: 248-50$.

13. Chelbi-Alix MK, Quignon F, Pelicano L, et al. Resistance to virus infection conferred by the interferon-induced promyelocytic leukemia protein. J Virol $1998 ; 72$ : 1043-51.

14. Regad T, Saib A, Lallemand-Breitenbach V, et al. PML mediates the interferon-induced antiviral state against a complex retrovirus via its association with the viral transactivator. EMBO J $2001 ; 20$ : 3495-505.

15. Pampin M, Simonin Y, Blondel B, et al. Cross talk between PML and $p 53$ during poliovirus infection: implications for antiviral defense. J Virol 2006 ; $80: 8582-92$

16. Blondel $\mathrm{D}$, Kheddache $\mathrm{S}$, Lahaye $\mathrm{X}$, et al. Resistance to rabies virus infection conferred by the PMLIV isoform. J Virol $2010 ; 84: 10719-26$.

17. Maroui MA, Pampin M, Chelbi-Alix MK. Promyelocytic leukemia isoform IV confers resistance to encephalomyocarditis virus via the sequestration of 3D polymerase in nuclear bodies. J Virol $2011 ; 85$ : 13164-73.

18. દા Asmi F, Maroui MA, Dutrieux J, et al. Implication of PMLIV in both intrinsic and innate immunity. PLoS Pathog $2014 ; 10$ : e1003975.

19. Regad T, Chelbi-Alix MK. Role and fate of PML nuclear bodies in response to interferon and viral infections. Oncogene $2001 ; 20$ : 7274-86.

20. Everett RD, Chelbi-Alix MK. PML and PML nuclear bodies: implications in antiviral defence. Biochimie 2007 ; 89 : 819-30.

21. Geoffroy MC, Chelbi-Alix MK. Role of promyelocytic leukemia protein in host antiviral defense. J Interferon Cytokine Res $2011 ; 31$ : 145-58.

22. Chelbi-Alix MK, Wietzerbin J. Interferon, a growing cytokine family: 50 years of interferon research. Biochimie $2007 ; 89: 713-8$.

23. Chelbi-Alix MK, Pelicano L, Quignon F, et al. Induction of the PML protein by interferons in normal and APL cells. Leukemia $1995 ; 9$ : 2027-33.

24. Stadler M, Chelbi-Alix MK, Koken MH, et al. Transcriptional induction of the PML growth suppressor gene by interferons is mediated through an ISRE and a GAS element. Oncogene $1995 ; 11: 2565-73$. 


\section{RÉFÉRENCES}

25. El Bougrini J, Dianoux L, Chelbi-Alix MK. PML positively regulates interferon gamma signaling. Biochimie $2011 ; 93: 389-98$

26. Bonilla WV, Pinschewer DD, Klenerman $P$, et al. Effects of promyelocytic leukemia protein on virushost balance. J Virol $2002 ; 76: 3810-8$.

27. McNally BA, Trgovcich J, Maul GG, et al. A role for cytoplasmic PML in cellular resistance to viral infection. PloS one $2008 ; 3$ : e2277.

28. Reichelt M, Wang L, Sommer M, et al. Entrapment of viral capsids in nuclear PML cages is an intrinsic antiviral host defense against varicella-zoster virus. PLoS Pathog $2011 ; 7$ : el001266.

29. Cuchet $D$, Sykes A, Nicolas A, et al. PML isoforms I and II participate in PML-dependent restriction of HSV-1 replication. J Cell Sci $2011 ; 124: 280-91$.

30. Mitchell AM, Hirsch ML, Li C, Samulski RJ. Promyelocytic leukemia protein is a cell-intrinsic factor inhibiting parvovirus DNA replication. J Virol $2014 ; 88$ : 925-36.

31. Aminev AG, Amineva SP, Palmenberg AC. Encephalomyocarditis virus (EMCV) proteins $2 A$ and $3 B C D$ localize to nuclei and inhibit cellular mRNA transcription but not rRNA transcription. Virus Res $2003 ; 95: 59-73$.

32. El Mchichi B, Regad T, Maroui MA, et al. SUMOylation promotes PML degradation during encephalomyocarditis virus infection. J Virol $2010 ; 84: 11634-45$.

33. Kyratsous CA, Silverstein SJ. Components of nuclear domain 10 bodies regulate varicella-zoster virus replication. J Virol $2009 ; 83: 4262-74$.

34. Yaffe MB, Schutkowski M, Shen M, et al. Sequence-specific and phosphorylation-dependent proline isomerization: a potential mitotic regulatory mechanism. Science 1997 ; 278 : 1957-60.
35. Liou YC, Zhou XZ, Lu KP. Prolyl isomerase Pinl as a molecular switch to determine the fate of phosphoproteins. Trends Biochem Sci 2011 ; 36 : 501-14.

36. Saitoh T, Tun-Kyi A, Ryo A, et al. Negative regulation of interferonregulatory factor 3 -dependent innate antiviral response by the prolyl isomerase Pinl. Nat Immunol 2006 ; 7 : 598-605.

37. Yang K, Shi HX, Liu XY, et al. TRIM21 is essential to sustain IFN regulatory factor 3 activation during antiviral response. J Immunol 2009; 182 : 3782-92.

38. Imler JL, Ferrandon D. Le printemps de l'immunité innée couronné à Stockholm. Med Sci (Paris) $2001 ; 27: 1019-24$

39. Jemilloux $Y$, Henry T. Les inflammasomes. Plates-formes de l'immunité innée. Med Sci (Paris) 2013 ; 29 : 975-84.

40. Marsolier J, Weitzman JB. Pin l : une peptidyl prolyl cis-trans isomérase multifonctionnelle et une cible anticancéreuse prometteuse. Med Sci (Paris) $2014 ; 30: 772-8$.

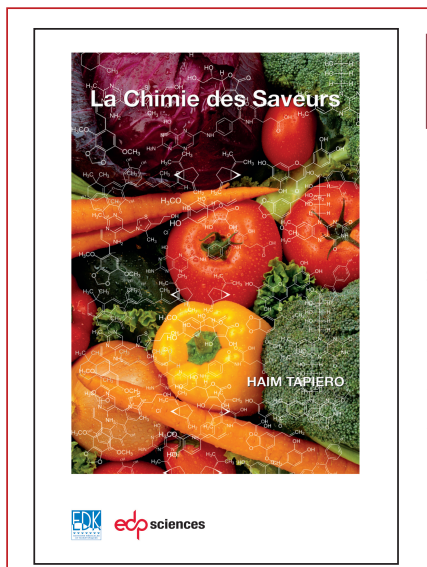

ISBN : 978-2-7598-1137-3 180 pages a cuisine est une science. II existe une relation étroite entre élaborer une recette et entreprendre une recherche scientifique. Quelle que soit l'origine d'une recette, d'un livre ou inventée, il faudra faire le choix des ingrédients, les mélanger et les cuire de manière appropriée afin de ne pas altérer les substances actives qui composent les ingrédients.

Une fois la cuisson terminée, il faudra analyser le goût et si nécessaire prévoir son amélioration. Améliorer une recette nécessite de connaître le ou les processus qui interviennent dans le développement des arômes, des saveurs et de la texture. Cette approche est similaire à celle développée par le scientifique.

La relation entre l'élaboration des recettes, les substances nutritives qui composent les ingrédients et la santé de l'homme est issue de plusieurs disciplines de la recherche fondamentale et clinique. Au cours des dernières années, de nombreux travaux scientifiques ont été publiés sur le rôle de la nutrition et la réduction des risques dans les pathologies comme les maladies cardio-vasculaires ou les cancers.

Le but principal de cet ouvrage a été d'identifier la structure chimique des composants actifs des ingrédients utilisés en cuisine (légumes, herbes aromatiques, épices) et qui entrent dans la préparation des recettes pour « végétariens » et « omnivores ».

\section{$--8<$}

À retourner à EDK, 109, avenue Aristide Briand - 92541 Montrouge Cedex

U Tél. : 0141177405 - Fax : 0141177405 - E-mail : edk@edk.fr

NOM : Prénom :

Adresse :

Code postal :

Pays :

Fonction :

Je souhaite recevoir l'ouvrage La chimie des Saveurs : $20 €+3 €$ de port $=\mathbf{2 3} €$ TTC

en ................ exemplaire, soit un total de .

$\ldots$

U Par chèque, à l'ordre de $\mathbf{E} \mathbf{D}$ K

- $\square$ Par carte bancaire : $\square$ Visa $\square$ Eurocard/Mastercard

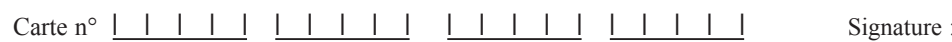

Date d'expiration: $\quad$ । 1 | 1 |

$\mathrm{N}^{\circ}$ de contrôle au dos de la carte :

Ville :

TIRÉS À PART

S. Nisole

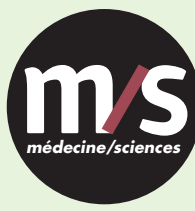

Tarifs d'abonnement $\mathrm{m} / \mathrm{s}-2014$

Abonnez-vous

à médecine/sciences
$>$ Grâce à $m / s$, vivez en direct les progrès des sciences biologiques et médicales

Bulletin d'abonnement page 813 dans ce numéro de $\mathrm{m} / \mathrm{s}$

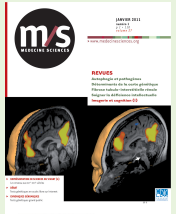

